\title{
1 Absolute quantitation of serum antibody reactivity using the Richards growth model for
}

4 Krisztián Papp, Ágnes Kovács, Anita Orosz, Zoltán Hérincs, Judit Randek, Károly Liliom,

5 Tamás Pfeil, József Prechl

7 Author information

8 These authors contributed equally: Krisztián Papp, Ágnes Kovács, Anita Orosz

R\&D Laboratory, Diagnosticum Zrt, Budapest, Hungary

11 Krisztián Papp, Zoltán Hérincs, József Prechl

12 Department of Applied Analysis and Computational Mathematics, Eötvös Loránd University, 13 Budapest, Hungary

14 Ágnes Kovács, Tamás Pfeil

15 Department of Immunology, Eötvös Loránd University, Budapest, Hungary

16 Anita Orosz

17 BME, Budapest University of Technology and Economics, Budapest, Hungary

18 Judit Randek

19 Department of Biophysics and Radiation Biology, Semmelweis University, Budapest, Hungary

20 Károly Liliom

21

22 Corresponding author: József Prechl,jprechl@diagnosticum.hu

1047 Attila út 126. Budapest, Hungary; T: +36-1-8810161

24

Current addresses

26

Anita Orosz : Department of Physiology, Semmelweis University, Budapest, Hungary;

27 Judit Randek : F. Hoffmann - La Roche Ltd., Basel, Switzerland 


\section{Abstract}

30 In spite of its pivotal role in the characterization of humoral immunity, there is no accepted 31 method for the absolute quantitation of antigen specific serum antibodies.

32 We devised a novel method to quantify polyclonal antibody reactivity, which exploits protein 33 microspot assays and employs a novel analytical approach. Microarrays with a density series 34 of disease specific antigen were treated with different serum dilutions and developed for IgG 35 and IgA binding. By fitting binding data of both dilution series to a product of two generalized 36 logistic functions, we obtained estimates of antibody reactivity of two immunoglobulin classes 37 simultaneously. These estimates are the antigen concentrations required for reaching the inflection point of thermodynamic activity coefficient of antibodies and the limiting activity coefficient of antigen.

40 By providing universal chemical units, this method may improve standardization of serological 41 testing, the quality control of antibodies and the quantitative mapping of antibody-antigen 42 interaction space.

Keywords:

46 polyclonal, antibody, quantitative, serology, immunoglobulin, antigen, immune response, 47 chemical potential, activity coefficient, microarray, Richards curve 


\section{Introduction}

Antibodies are glycoproteins belonging to the immunoglobulin protein superfamily, produced by B cells of the adaptive immune system. Antibodies in an organism are structurally highly heterogeneous owing to the gene recombination-mediated diversity of the adaptive immune response and the clonal nature of B-cell expansions ${ }^{1,2}$. A single given B cell and its progeny of plasmablasts and plasma cells produce antibody molecules with a given target specificity and binding affinity, determined by the fit of the rearranged heavy and light chain variable regions. Millions of B cells carrying different rearranged heavy and light chain variable regions generate a network of diverse antibodies present in an individual ${ }^{3}$. A humoral immune response against a particular antigen involves the evolution of antibody producing plasmablast and plasma cell clones with various isotypes and affinities, these latter properties also being characteristic for the polyclonal response besides target specificity. The affinity of an antibody molecule towards its target antigen is an important characteristic both from the biochemical and immunological point of view. Biochemically, it reflects the strength of interaction between the two binding partners ${ }^{4}$. Immunologically, it is closely correlated with effector functions such as neutralization efficiency and complement activation ${ }^{5}$. The isotype of the antibody molecule is the other key determinant of effector function. Understanding these properties of serum antibodies generated against particular antigenic targets is the goal of serological measurements, which aim at the laboratory diagnosis of infectious, allergic and autoimmune diseases ${ }^{6-8}$. Evidently, medical laboratory diagnostics requires the standardization of serological measurements.

Standardization of the measurement of antibody reactivity has always been a difficult issue ${ }^{9}$ and in spite of much effort it has not been fully resolved ${ }^{10}$. Among the analytical issues is the fact that the quantitation of a heterogeneous population of antibodies would require a heterogenous reference material. Currently this is achieved by the utilization of affinity purified polyclonal serum pools or pools of monoclonal antibodies. These reagents, along with the standardization and harmonization of measurement protocols led to current best laboratory practices, which provide results in international units. However, quantitative comparison of different assays with different units is not meaningful, therefore systems level quantitative representation of antibody reactivity is currently impossible.

Determination of antibody affinity has always been considered of key importance for the characterization of humoral immunity. From the immunochemical and biophysical point of view, the techniques employed range from equilibrium dialysis ${ }^{11}$, precipitation assays using radiolabeling ${ }^{11}$, use of denaturants to disrupt binding forces ${ }^{12}$, setting up competition assays 
13 and varying $\mathrm{Ag}$ density in enzyme-linked immunosorbent-assays (ELISA) ${ }^{14}$. A noncompetitive ELISA ${ }^{15}$ for the measurement of monoclonal antibody affinity that relies on the determination of $\mathrm{Ab}$ binding at different antigen coating densities shares some conceptual basis with our assay, however it requires the use of known concentrations of monoclonal antibodies. Alternatively, equilibrium binding constants can be obtained from kinetics measurements by surface plasmon resonance analysis ${ }^{16}$, biolayer interferometry ${ }^{17}$, microscale thermophoresis 18 and similar technologies. While these measurements are often regarded as the golden standard of affinity determination, the instrumentation and data-intensive nature of measurement may not be optimal for routine diagnostic use. Additionally, label-free methods are not easily adaptable for the selective measurement of antibody classes, a must for immunodiagnostics.

Serum antibody titration is a simple and widely used way of estimating antibody reactivity, wherein the sample is serially diluted and the highest dilution that gives a signal reliably discernible from the background, or the dilution resulting in half signal, are regarded as the titer for endpoint and midpoint titration, respectively. Such titers may provide fast and costeffective semi-quantitative results but are not suitable for real quantitation of serum antibodies because we are changing an unknown variable when we decrease antibody concentration by serum dilution.

The unique properties of microspot immunoassays were described by Ekins ${ }^{19}$, who propagated their use for capture immunoassays ${ }^{20}$. The general idea is that owing to the negligible amount of molecules present in the microspot probe compared to the amount of analyte in the tested solution, these assays are mass independent ${ }^{20}$ : interactions are governed by concentration and affinity only. When affinity is constant and homogenous, as in a capture immunoassay utilizing monoclonal antibody, the density of analyte bound to the surface is determined solely by its concentration. Here we propose that mass independence of microspot immunoassays in fact requires an analytical approach that is different from conventional calculations based on the law of mass action ${ }^{15,21}$, which applies to reactions in solutions. We suggest the use of concepts and terminology of physical chemistry, which are readily applicable to reactions at a solidliquid interface ${ }^{22,23}$.

In this paper we present a microspot-based approach that allows the estimation of apparent chemical potential of distinct antibody isotypes in human serum without any prior purification steps. The measurement relies on the simultaneous titration of antigen surface density and serum dilution and on an improved mathematical model of fitting. Most importantly, by changing a known variable of the system, antigen concentration, the technology allows the 
119 absolute quantification of antigen specific reactivity of polyclonal serum antibodies, allowing quantitative comparison of different samples, isotypes or antigens.

\section{Results}

\section{Experimental setup and properties of the measurement system}

124 The experimental system we use is characteristic of protein microarray technology with some 125 key differences to traditional indirect ELISA. The solid surface used as antigen adsorbent has higher binding capacity than conventional polystyrene. This results in a dose dependent adsorptive binding over a wider concentration range. Therefore, the concentration of antigen in the solution used for bio-printing (spotting of antigen) is expected to show linear relationship with the surface density of the antigen on the microarray over a correspondingly wider range. Antibodies that bind to the spotted antigens are detected by fluorescently labelled secondary antibodies. Owing to the properties of fluorescent measurement, bound antibodies can be detected over a wide range as well. To allow for the analysis and visualization of signals spreading over several orders of magnitude, the logarithm of both antigen concentrations and fluorescent signal intensities is used.

To establish a distribution of immune complex concentrations as a function of antigen density we employed two different, complementary strategies (Fig. 1). First, increasing concentrations of antigen $[\mathrm{Ag}]$ were printed on a solid surface as microspots. Owing to the negligible amount of antigen in a microspot compared to the amount of antibody in the reaction solution, these measurement conditions are called mass-independent or ambient analyte immunoassay ${ }^{19,20}$.

140 Antigen microspots therefore probe antibody reactivity in the sample without significantly 141 altering composition of the sample. Second, dilutions of the serum samples of interest were 142 prepared to vary the concentration of antibody-antigen complexes [AbAg] in the measurement 143 range and to examine the relationship between antibody concentration $[\mathrm{Ab}]$ and $[\mathrm{AbAg}]$. Even

144 though the absolute free antibody concentration $[\mathrm{Ab}]$ in the tested serum is not known, the 145 relative concentrations of the dilution series can be used to follow [Ab] effects by mathematical 146 curve fitting. Dilutions of serum samples were applied to the series of antigen density microspots and incubated to bring the system in equilibrium. 
151 The resulting measurement system examines the effect of changes in relative $[\mathrm{Ab}]$ and $[\mathrm{Ag}]$ on

152

153

154

155

156

157

158

159

160

161

162

163

164

165

166

167

168

169

170

171

172

173

174

175

176

177

178

179

180

181 the extent of $\mathrm{Ab}$ binding. The obtained experimental data are fitted with a growth function to derive values of the parameters that characterize antibody reactivity in the sample (Fig. 1).

\section{Curve fitting}

The formation of AbAg complexes and the extent of antibody binding under equilibrium in our assay can be interpreted as the indicator of $\mathrm{Ab}$ thermodynamic activity in the microspots. The equilibrium concentration of $\mathrm{AbAg}$ complexes is a logistic function of $\ln [\mathrm{Ag}]$, and the logarithm of the fluorescent intensity is a linear function of $\ln [\mathrm{AbAg}]$, therefore the growth of fluorescent intensity can be described by the generalized logistic function ${ }^{24,25}$ or Richards function $\mathrm{R}(\mathrm{x})$ of $\mathrm{x}=\ln [\mathrm{Ag}]$, an extended form of the function most frequently used for immunoassays. We use the following parametrization

$R(x)=A *\left(1+(d-1) e^{-k *\left(x-x_{i}\right)}\right)^{\frac{1}{1-d}}$

where $\mathrm{A}$ is $\mathrm{Ab}$ capacity, $[\mathrm{Ab}]$; $\mathrm{k}$ is rate of exponential growth; $\mathrm{d}$ determines asymmetry, the relative value of $\mathrm{R}$ at fastest growth; $\mathrm{x}_{\mathrm{i}}$ is the point of fastest growth or inflection point.

The capacity of the system is determined by the availability of antibody, [Ab]. While the exact value is unknown, relative values corresponding to steps in dilution series can be used in the fitting procedure. In our assay exponential growth of $[\mathrm{AbAg}]$ is allowed by the provision of exponentially increasing $[\mathrm{Ag}]$ in a series of microspots. We assume that antigen titration results in a binding curve following a generalized logistic curve with the rate of exponential growth $\mathrm{k}=1$, which corresponds to $[\mathrm{Ag}]=\mathrm{e}^{1 * \ln [\mathrm{Ag}]}$. Shape parameter $\mathrm{d}$ of the generalized logistic function allows for asymmetry in the binding curve. In our assay $\mathrm{d}$ is an index, which characterizes the $\mathrm{Ab}$ composition of serum. Location of the fastest growth $\mathrm{x}_{\mathrm{i}}$ with respect to $\ln [\mathrm{Ag}]$ provides a general measure of antibody affinity.

Affinities and concentrations of serum antibodies are distributed over a very wide range, equilibrium dissociation constants from $10^{-5}$ to $10^{-11}$ and molar concentrations from $\mathrm{pM}$ to $\mathrm{nM}$, respectively. In order to better assess interactions over this range and to weigh curve fitting against signal intensity-dependent variation, we use logarithmically transformed signals of binding. Thus, fitting the Richards curve to our measurements therefore requires the logarithmic form of the above equation

$\ln R(x)=\ln (A)+\frac{1}{1-d} * \ln \left(1+(d-1) * e^{-k *\left(x-x_{i}\right)}\right)$ 
182

By using the logarithm we transform thermodynamic activity to chemical potential of the $\mathrm{Ab}$, as determined by its standard molar Gibbs free energy, concentration and activity coefficient

$\mu_{A b}=\mu^{\circ}+R T \ln \left(a_{A b}\right)=\mu^{\circ}+R T \ln \left(c_{A b} * \gamma_{A b}\right)=\mu^{\circ}+R T \ln \left(c_{A b}\right)+R T \ln \left(\gamma_{A b}\right)$

where $\mu$ is chemical potential; $\mu^{\circ}$ is standard chemical potential or molar Gibbs free energy; $a$ is relative thermodynamic activity; $\gamma$ is activity coefficient; and $\mathrm{c}$ is molar concentration. Since the standard term serves as a reference point, we can still obtain relative potentials after its removal. Then we can interpret $\ln (\mathrm{A})$ of the $\ln \mathrm{R}(\mathrm{x})$ function as the mole fraction term of chemical potential and the remaining part of the function describes the activity coefficient as a function of $[\mathrm{Ag}]$ (for details see Supplementary text 1).

To demonstrate the dependence of $\ln (\mathrm{A})$ from the serum dilution we extended the formula of $\ln R(x)$ to obtain the combined fitting of serum dilutions and antigen dilutions:

$\ln _{2}(x, z)=\ln (C)-m * \ln (B+z)+\frac{1}{1-d} * \ln \left(1+(d-1) * e^{-\left(x-x_{i}\right)}\right)$

where $\mathrm{z}$ is serum dilution, and $\mathrm{m}, \mathrm{B}, \mathrm{C}$ are auxiliary parameters of the logarithm of another generalized logistic function(Tjørve and Tjørve 2010) of $-\ln (\mathrm{z})$ that replaced $\ln (\mathrm{A})$ and has a parametrization different from formula $(2$.

Reliable curve fitting depends on the number and location of $x=\ln [\mathrm{Ag}]$ measurement points on the curve and requires the value of $x_{i}$ to be in the measurement range.

\section{Characterization of anti-deamidated gliadin peptide serum antibodies}

As an exemplary antigen for the proof of our measurement principle we choose a deamidated peptide sequence known to be the target of antibodies in celiac disease. Such an antigenic peptide is a well-defined molecular target of both IgA and IgG antibodies in celiac patients and is used in laboratory diagnostics. We used peptide concentrations corresponding to the range of $K_{D}$ values expected to occur in serum and peptide dilutions that well extended beyond that range. Serum samples were diluted to span about two orders of magnitude and correspond to dilutions conventionally used in serological diagnostics. Binding data were fitted using the $\ln R_{2}(x)$ function of equation (4 introduced above and generated curves were overlain on the binding data (Fig. 2)

\section{Figure 2. Fitting in two dimensions}


213 Using the values of parameters from the fitted binding curves of experimental data, we can

214 generate binding curves normalized to Ab concentrations, which are curves of thermodynamic

215 activity coefficients $\gamma_{A b}$, as illustrated in Figure 3.

216

217 Figure 3. Comparative characterization of serum antibody binding

219 These curves are independent of antibody concentrations and fluorescent intensity and are therefore comparable for different classes of $\mathrm{Ab}$ in a given sample, for different samples and any combination thereof. Parameter $\mathrm{x}_{\mathrm{i}}$ and $\gamma_{A b}$ are sufficient to quantitatively characterize the distribution of $\mathrm{Ab}$ thermodynamic activity in the tested serum sample as long as the Richards curve models binding events.

224 To confirm that conventional titration cannot provide exact results we calculated titers from

225 the binding data obtained from the microspot experiments. The classical approach of serum titration was heavily dependent on the antigen density, as observed by others ${ }^{13,26,27}$. We calculated end-point titers and mid-point titers of the same measurements by logistic fitting of binding curves to distinct antigen densities, and obtained different titers for different antigen densities (Fig. 4). This antigen density dependence is not only avoided but is instead exploited in our approach of fitting curves to antigen density.

Figure 4. Conventional titration of serum

234 We also confirmed the applicability of our method to other antigens using a microarray printed with dilution series of citrullinated peptides known to be targets of autoantibodies of rheumatoid arthritis patients (Supplementary Fig. S1).

Measurement of reference monoclonal antibody properties

239 Current serological assays use standardized antibody preparations as reference material for quality control and calibration ${ }^{10}$. These preparations are monoclonal antibodies alone or in combinations of monoclonal antibodies, or pooled raw or isolated serum antibodies from positive samples. Because of the individual variance of affinity distribution of serum antibodies these approaches have the major drawback of assuming identical affinity distribution of reference and test samples. Titration by antigen density avoids this pitfall since it does not a priori assume but it rather identifies distribution of samples and reference standards. The 
critical point of antigen density titration is the precise deposition of $\mathrm{Ag}$ on the microarray surface, so that the real density of antigen corresponds to the nominal antigen concentration used for printing. The precision can be estimated by measuring and characterizing a reference antibody. We successfully used the monoclonal antibody 14D15 specific for an epitope in our deamidated gliadin peptide sequence to characterize their interaction (Fig. 5).

Figure 5. Characterization of a monoclonal $A b$

\section{Discussion}

256 The concentrations of serum antibodies and of targets of these antibodies, along with the range of the strength of these interactions span several orders of magnitude. To obtain a systems level structured map of these interactions we need technologies that provide quantitative results of molecular interaction measurements over this wide range, from low picomolar to micromolar concentrations. Here we present results, which together with earlier observations ${ }^{28,29}$ provide evidence and theoretical support for protein microspot-based fluorescent detection as a method of choice.

The difficulty of rendering the measurement of polyclonal antibody responses quantitative lies partly in the reproducible generation of rigorously characterized antigen, partly in the mathematical description of heterogeneity of polyclonal antibody response. The generation of synthetic epitopes for serological assays, along with functional characterization using reference affinity reagents may solve the first issue ${ }^{10,30}$. Our approach may provide a solution for the second one: establishing standardized assays that generate quantitative data with SI units of measurement. The key for generating a quantitative description of polyclonal serum antibody reactivity is the ability to dissect antibody concentration and affinity, the two parameters that determine antigen saturation. This is basically achieved by titrating both concentration and affinity: in addition to the conventional serum dilution series, a series of antigen dilutions is used concomitantly. By diluting serum antibody, a series of antibody concentrations is examined, while by additionally using microspots of antigen dilution series, affinity distribution is assessed simultaneously. Microspots allow a mass-independent interrogation of interactions on the chip surface, whereby the measurement becomes independent of the relative total masses of $\mathrm{Ag}$ and $\mathrm{Ab}$ in the reaction volume. Complex formation is determined only by concentration and affinity, which two measures can be deconvolved by two-dimensional titration. By gradually increasing the Ag density of the surface, an Ab with a given affinity will engage in lower affinity interactions as well. This results in the measurement of a range of 
281 affinities from the highest (detected on low density Ag spots) to more degenerate lower affinity interactions.

283 There have been several attempts to characterize the affinity of antibodies associated with autoimmunity ${ }^{31-34}$, infectious disease ${ }^{35,36}$, vaccination ${ }^{37}$ and allergy ${ }^{14,38-41}$. The strength of binding of serum antibodies is determined by the distribution of clones with different affinities specific for the tested epitope. While the importance of antibody affinity in conferring pathogenicity or protectivity is widely acknowledged, affinity determination is not generally considered as part of diagnostic serology. The possible reason is that affinity measurement is technologically challenging and is not standardized, as outlined above. Affinity dependence of traditional immunoassays (e.g., RIA, ELISA, Farr assay, hemagglutination, complementmediated haemolysis, precipitation) ${ }^{42}$ practically excludes low affinity interactions from measurement and may skew affinity determinations when such assays are used. Such a progressive decrease of sensitivity to low affinity antibodies should lead to estimations of antibody affinity distribution with a higher mean affinity. The method we propose here presumes the existence of low affinity antibodies and measures them by using Ag at very high densities as well.

Microspot immunoassays were introduced for the measurement of analyte concentration, taking advantage of the very low amount of capture antibody required by the technology. If in the complete volume of measurement, the concentration of antibody on the solid support is less than $1 \%$ of the $\mathrm{K}_{\mathrm{D}}$ of interaction, then the concentration of the analyte does not change significantly $(<1 \%)$ during the measurement. The signal from a detection antibody is therefore correlated only with analyte concentration when other variables are kept constant, which conditions are characteristic of ambient analyte immunoassay ${ }^{19}$. The analyte in our assay is serum antibody and the $\mathrm{K}_{\mathrm{D}}$ of interactions are heterogeneous. Nevertheless, the amount of antigen and therefore its concentration in the total measurement volume is still negligible. Our method takes further advantage of microspots by varying the surface density of antigen for antibody capture. Since the relative concentrations of antibodies with different affinities are not changed by serum dilution, the affinity profile of antibodies that make contact with antigen on the solid support does not change either. The activity coefficient of this population of antibodies will depend solely on the density of antigen $[\mathrm{Ag}]$ relative to the average affinity of interactions. By varying $[\mathrm{Ag}]$ we obtain a distribution curve of chemical potentials suitable for estimating values of the parameters of fitted function.

313 The interpretation of Richards curve parameters provides insight into several aspects of serum antibody reactivity. Parameter A is related to the molar concentration of functional antibody 
binding sites, called paratopes, belonging to the measured immunoglobulin class. Because the measurement of distribution of affinity reaches into the low affinity range, the estimated concentrations are approximations of total serum antibody concentrations. We expect this value to be dependent and correlated with serum antibody concentrations and therefore to have less diagnostic value. Thus, it is presumably not necessary to adjust for antibody valency and convert binding site concentrations into isotype concentrations. Antibody responses that exploit the increase of antibody concentrations without major increase in affinity, like the thymusindependent response, are therefore expected to appear as a change in this parameter. A technical advantage of rendering the binding parameters independent of total serum antibody concentrations is that we also exclude absolute fluorescence intensity values from the analysis, thus removing a factor of interlaboratory variance.

The central point or inflection point $\mathrm{x}_{\mathrm{i}}$ is related to the apparent chemical potential of serum antibodies. We propose to use the short name "lnK $\mathrm{D}$ " for the natural logarithm of molar concentration of antigen required to reach maximal relative growth of antibody activity coefficient $\gamma_{\mathrm{Ab}}$ (inflection point of sigmoid curve in log-lin scale). While the unit of chemical potential is that of energy (Joule/mole) here we would retain the unitless number derived from antigen concentration titration.

The Richards curve uses parameter $d$ to introduce asymmetry on the two sides of the inflection point. At $d=2$ the curve is symmetric and it is a logistic curve. At $d \rightarrow 1$ the curve approaches the Gompertz growth curve and becomes asymmetric as the inflection point shifts from $\mathrm{y}_{\mathrm{i}}=\mathrm{A} / 2$ to $\mathrm{y}_{\mathrm{i}}=\mathrm{A} / \mathrm{e}$.

The slope of the $\operatorname{lnR}_{2}(\mathrm{x})$ function $\mathrm{s}=\mathrm{k} /(\mathrm{d}-1)$ at minus infinity, in the case $\mathrm{k}=1$, meanwhile increases from $s=1$ to $\infty$. This slope characterizes interactions as antigen is diluted out to infinity. Infinite dilution is a special thermodynamic state when antigen molecules are in contact with antibody molecules only ${ }^{43}$. In this state binding is determined only by interactions between $\mathrm{Ag}$ and $\mathrm{Ab}$, without the interference of homotypic interactions. This ideal state is characterized by the limiting activity coefficient $\gamma_{i}^{\infty}$. Using our parametrization d-1 changed between 0 and 1 . We propose that $\mathrm{d}-1$ is the limiting activity coefficient of $\mathrm{Ag}, \gamma_{A g}^{\infty}$. It characterizes the composition of $\mathrm{Ab}$ mixture with 0 representing no interacting $\mathrm{Abs}$ and 1 representing 100\% presence of binding Abs at infinite Ag dilution. We expect that parameter $\mathrm{d}$ can be used to characterize disease activity when antibody diversity and affinity is related to disease pathogenesis, with lower $\mathrm{d}$ implying immunological activity, and higher $\mathrm{d}$ indicating approach of equilibrium concentrations and stability of the immune response. 
348 A potential disadvantage of the proposed method is that it is technologically challenging, 349 compared to established automated assays. A critical point is the generation of antigen spots with real densities as close to nominal densities as possible. This requires the introduction of extra quality control steps into the production. The fitting procedure requires a minimal number of data points around the estimated inflection point, therefore weakly reactive samples cannot be quantitated. These disadvantages position our assay as a fine analysis method, suitable not for the screening and identification of presence of specific antibodies but rather for the detailed characterization of pre-screened sera.

A key conceptual novelty in our assay is that instead of assessing the concentration of a heterogeneous antibody population we assess distribution of apparent chemical potential. Current immunoassays focus on establishing conditions that are ideal for estimation of the concentration of antibodies against a particular target antigen. For solid phase assays this involves the optimization of coating antigen density, where actually an antigen density suitable for identifying the shift of average $\mathrm{K}_{\mathrm{D}}$ in a diagnostically relevant region of affinity is identified. By using microspots with antigen densities spanning the whole range of relevant concentrations, a single measurement can provide a distribution of apparent $\mathrm{Ab}$ chemical potentials.

The procedure we describe here assumes that equilibrium is reached when microspots are incubated in serum. In order to confirm that measurement conditions are appropriate and to allow reproducibility and interassay comparisons, antibody standards can be introduced into the assay. Pooled positive and borderline serum samples could be used in our assay just as in any other immunoassay for specific antibody measurement. Additionally, monoclonal antibody preparations are also suitable as internal reference, since calculations of sample Ab affinity would not be based on the binding of reference antibody, but these would rather serve as controls for validation and perhaps for adjusting calculations.

373 The introduction of such quantitative assays could not only improve diagnostic accuracy of 374 immunoassays but would also pave the way to our quantitative understanding of the adaptive immune system. The generalized quantitative model of antibody homeostasis ${ }^{3}$ provides a conceptual framework for the experimental mapping of the "antibodyome" 44-46, a goal that could be achieved by the strategic mapping of antigen structural space. The integration of quantitative binding information into a network and the mapping of the interaction network ${ }^{45}$ to the sequence space generated by next generation sequencing ${ }^{47}$ is expected to bring about unprecedented systemic understanding of adaptive immunity in particular and protein evolution in general. 


\section{Materials and methods}

\section{Microarray production and measurements}

385 Experiments were carried out on hydrogel coated glass slides (Nexterion Slide H) by using a BioOdyssey Calligrapher MiniArrayer. Different peptide dilutions, as indicated in the text, were spotted in quintuplicates of $1 / 2$ serial dilution in 7 steps starting from $200 \mu \mathrm{M}$. Slides were dried for $1 \mathrm{~h}$ at $37^{\circ} \mathrm{C}$ then soaked in $0.1 \mathrm{M}$ Tris buffer $(\mathrm{pH}=8)$ for $1 \mathrm{~h}$ at $37^{\circ} \mathrm{C}$ in order to block reactive residues on the surface. Once prepared, slides were kept in sealed non-transparent bags at $4{ }^{\circ} \mathrm{C}$. The fusion peptide GLN1-2F was based on the deamidated gliadin sequence motifs LQPFPQPELPYPQPQ and PLQPEQPFP.

Previously characterized, frozen serum samples collected for studies with contract numbers: 24933-6/2018/EKU and 24973-1/2012/EKU (658/PI/2012.) were used for the experiments. Those studies were aimed at developing serological assays for gluten sensitivity and autoimmune diseases, respectively, and all procedures were in accordance with the ethical standards of the responsible committee on human experimentation and with the Helsinki declaration of 1975 , as revised in 2008.

398

\section{Sample handling and signal detection}

400 The basic protocol was the following: dried arrays were rehydrated in $110 \mu 1 \mathrm{PBS}(3 \times 5 \mathrm{~min})$ 401 before using, then sub-arrays were incubated in $70 \mu \mathrm{l}$ diluted sample at $37^{\circ} \mathrm{C}$ for $1 \mathrm{~h}$. Sample 402 dilution was carried out in PBS-BSA-Tween (PBS, 2\% BSA, 0.05\% Tween 20). Serum treated 403 slides were washed in $0.05 \%$ Tween-PBS, then incubated at room temperature for 30 minutes with fluorescently labelled antibodies that were diluted in the blocking buffer $(0.05 \%$ Tween 20, 2\% BSA, PBS). Detecting antibodies were the following: Fc $\gamma$ fragment specific DyLight 649-conjugated AffinityPure F(ab')2 fragment goat anti-human IgG; DyLight 549-conjugated AffinityPure F(ab')2 fragment goat anti human-IgA. Chips were washed again and following drying, slides were scanned using laser microarray scanner.

\section{Measurements with 14D5 monoclonal antibody}

411 The previously described basic protocol was altered for the assays with monoclonal antibody 412 against deamidated gliadin (14D5). First, slides were rehydrated in PBS $(3 \times 5 \mathrm{~min})$ then blocked 413 in blocking buffer $\left(0.05 \%\right.$ Tween $20,2 \%$ BSA, PBS) for 30 minutes at $37^{\circ} \mathrm{C}$. A dilution series 414 was prepared from the monoclonal antibody starting from $200 \mu \mathrm{g} / \mathrm{ml}$ by $1 / 2$ in 10 steps. 415 Detection was carried out by goat anti-mouse IgG2a-Alexa488. 


\section{Analysis of the microarray data}

418 Images of the slides were analyzed with GenePix Pro 6.0 software after visual inspection. Spots 419 were recognized by the program, then gpr files containing the spot coordinates for individual 420 spots were created. Then $\mathrm{R}$ (version 3.5.2), a statistical programming environment, was used 421 to re-analyse 16-bit tiff images by using these coordinates in order to align the spots. Relative 422 fluorescence intensity (RFI) medians were calculated for each spot using $80 \%$ of the diameter of the circle shapes that were previously adjusted on the features by the software. In the next step, means of the 5 parallel spots were taken and values of the least concentration antigen spots (considered as background) were subtracted of all RFI values, thus providing the final signal intensities.

\section{Fitting of binding curves}

429 In the indirect assay, we take into consideration that a bound antibody inhibits nearby free antigens from forming complexes with other antibodies ${ }^{48,49}$. This inhibition makes the concentration of immune complexes a logistic function of the logarithm of the total antigen concentration:

434 where $\mathrm{D}$ is positive constant.

435 It is the nature of our measurement system that the logarithm fluorescent intensity is a linear function of the logarithm concentration of $\mathrm{AbAg}$ complexes. By using these, we obtain that the logarithm of fluorescent intensity is a Richards function of the logarithm of total antigen concentration

$R(x)=A *\left(1+(d-1) * e^{-k\left(x-x_{i}\right)}\right)^{\frac{1}{1-d}}$

440 with $\mathrm{k}=1$, where $\mathrm{A}$ is the total antibody concentration [Ab] (limit of function $\mathrm{R}(\mathrm{x})$ at infinity),

$441 \quad \mathrm{x}_{\mathrm{i}}$ is the inflection point, $\mathrm{d}$ is the asymmetry parameter.

442 The upper limit of the fluorescent intensity A depends on the serum dilution. Formula (6) also 443 implies that the concentration of immune complexes is a logistic function of the logarithm of the total antibody concentration $\ln [\mathrm{Ab}]$ with growth rate 1, i.e. a logistic function of $-\ln (\mathrm{z})$ with growth rate 1 , where $\mathrm{z}$ is the serum dilution factor. Due to the linear dependence of the logarithm fluorescent intensity on the logarithm printed Ag concentration, the upper limit of the logarithm fluorescent intensity is a Richards function of the negative logarithm serum dilution $\mathrm{z}$ with growth rate 1 . 
449 Therefore, the fluorescent signal intensity against the logarithm total antigen concentration $\mathrm{x}$, 450 and the negative logarithm of serum dilution, $-\ln (\mathrm{z})$, is the product of two generalized logistic 451 functions of form

453 The logarithm transformation converts the proportional variance pattern to a constant variance 454 pattern and thus the conversion makes the transformed data more suitable for fitting the model. 455 The above multiplicative relationship now changes to an additive one in the form of

$456 \quad \ln R_{2}(x, z)=\ln (C)-m * \ln (B+z)+\frac{1}{1-d} * \ln \left(1+(d-1) * e^{-\left(x-x_{i}\right)}\right)$

457 This generalized logistic model on the log-log scale was fit to the data, and parameter estimates 458 and 95\% confidence intervals were obtained for each serum and both isotypes. All analyses 459 were carried out using the R software (version 3.5.2).

460 Nonlinear least squares estimates for the model parameters were calculated using the Gauss461 Newton algorithm of the nls function from the statistical software package R (version 3.5.2). 462 The 95\% confidence intervals generated for the model parameters were based on the profile 463 likelihood technique. 
466 1. Imkeller, K. \& Wardemann, H. Assessing human B cell repertoire diversity and convergence. Immunol. Rev. 284, 51-66 (2018).

2. Briney, B., Inderbitzin, A., Joyce, C. \& Burton, D. R. Commonality despite exceptional diversity in the baseline human antibody repertoire. Nature 566, 393-397 (2019).

3. Prechl, J. A generalized quantitative antibody homeostasis model: antigen saturation, natural antibodies and a quantitative antibody network. Clin. Transl. Immunology 6 ,

4. Marillet, S., Lefranc, M.-P., Boudinot, P. \& Cazals, F. Novel Structural Parameters of Ig-Ag Complexes Yield a Quantitative Description of Interaction Specificity and Binding Affinity. Front. Immunol. 8, 34 (2017).

5. Prechl, J. A generalized quantitative antibody homeostasis model: maintenance of global antibody equilibrium by effector functions. Clin. Transl. Immunology 6, e161 (2017).

479

6. Bacarese-Hamilton, T., Bistoni, F. \& Crisanti, A. Protein microarrays: from serodiagnosis to whole proteome scale analysis of the immune response against pathogenic microorganisms. BioTechniques Suppl, 24-29 (2002). serodiagnosis of rheumatoid arthritis. Rheumatol. Int. 36, 751-758 (2016).

8. Chokshi, N. Y. \& Sicherer, S. H. Interpreting IgE sensitization tests in food allergy. Expert Rev. Clin. Immunol. 12, 389-403 (2016). 
10. Meroni, P. L. et al. Standardization of autoantibody testing: a paradigm for serology in rheumatic diseases. Nat. Rev. Rheumatol. 10, 35-43 (2014).

11. Stanley, C., Lew, A. M. \& Steward, M. W. The measurement of antibody affinity: a comparison of five techniques utilizing a panel of monoclonal anti-DNP antibodies and the effect of high affinity antibody on the measurement of low affinity antibody. $\mathrm{J}$. Immunol. Methods 64, 119-132 (1983).

12. Macdonald, R. A., Hosking, C. S. \& Jones, C. L. The measurement of relative antibody affinity by ELISA using thiocyanate elution. J. Immunol. Methods 106, 191194 (1988).

13. Bruderer, U., Deusinger, M., Schürch, U. \& Lang, A. B. Analyses of affinity distributions within polyclonal populations of antigen-specific antibodies. Evaluation of their accuracy in population detection using monoclonal antibodies. J. Immunol. Methods 151, 157-164 (1992).

14. Nieto, A., Gaya, A., Jansa, M., Moreno, C. \& Vives, J. Direct measurement of antibody affinity distribution by hapten-inhibition enzyme immunoassay. Mol. Immunol. 21, 537-543 (1984).

15. Beatty, J. D., Beatty, B. G. \& Vlahos, W. G. Measurement of monoclonal antibody affinity by non-competitive enzyme immunoassay. J. Immunol. Methods 100, 173-179 (1987).

16. Sem, D. S., McNeeley, P. A. \& Linnik, M. D. Antibody affinities and relative titers in polyclonal populations: surface plasmon resonance analysis of anti-DNA antibodies. Arch. Biochem. Biophys. 372, 62-68 (1999).

17. Abdiche, Y., Malashock, D., Pinkerton, A. \& Pons, J. Determining kinetics and affinities of protein interactions using a parallel real-time label-free biosensor, the Octet. Anal. Biochem. 377, 209-217 (2008). 
513 18. Jerabek-Willemsen, M., Wienken, C. J., Braun, D., Baaske, P. \& Duhr, S. Molecular interaction studies using microscale thermophoresis. Assay Drug Dev. Technol. 9, 342-353 (2011).

19. Ekins, R., Chu, F. \& Biggart, E. Multispot, multianalyte, immunoassay. Ann Biol Clin (Paris) 48, 655-666 (1990).

20. Saviranta, P. et al. Evaluating sandwich immunoassays in microarray format in terms of the ambient analyte regime. Clin. Chem. 50, 1907-1920 (2004).

21. Larsson, A. Assessment of polyclonal antibody binding of ligand by Sips' equation or by the exact polyclonal equation. Comparison of models. Mol. Immunol. 25, 12391249 (1988).

523

22. Kallay, N., Preočanin, T., Kovačević, D., Lützenkirchen, J. \& Villalobos, M. Thermodynamics of the reactions at solid/liquid interfaces. Croat. Chem. Acta 1-10 (2011) doi:10.5562/cca1864.

23. Moradi, O. Thermodynamics of Interfaces. in Thermodynamics - Interaction Studies Solids, Liquids and Gases (ed. Moreno Pirajn, J. C.) (InTech, 2011). doi:10.5772/20083.

24. Gottschalk, P. G. \& Dunn, J. R. The five-parameter logistic: a characterization and comparison with the four-parameter logistic. Anal. Biochem. 343, 54-65 (2005).

25. Tsoularis, A. \& Wallace, J. Analysis of logistic growth models. Math. Biosci. 179, 2155 (2002).

26. Hadzhieva, M. et al. Impact of antigen density on the binding mechanism of igg antibodies. Sci. Rep. 7, 3767 (2017). analysed by monoclonal antibodies. J. Immunol. Methods 72, 171-176 (1984). 
537

538

539

540

541

542

543

544

545

546

547

548

549

550

551

552

553

554

555

556

557

558

559

560

28. Kusnezow, W. et al. Optimal design of microarray immunoassays to compensate for kinetic limitations: theory and experiment. Mol. Cell. Proteomics 5, 1681-1696 (2006).

29. Engelmann, B. W. et al. The development and application of a quantitative peptide microarray based approach to protein interaction domain specificity space. Mol. Cell. Proteomics 13, 3647-3662 (2014).

30. Rizwan, M. et al. Serology in the Digital Age: Using Long Synthetic Peptides Created from Nucleic Acid Sequences as Antigens in Microarrays. Microarrays (Basel) 5, (2016).

31. Achenbach, P. et al. Combined testing of antibody titer and affinity improves insulin autoantibody measurement: Diabetes Antibody Standardization Program. Clin. Immunol. 122, 85-90 (2007).

32. Szarka, E. et al. Affinity Purification and Comparative Biosensor Analysis of CitrullinePeptide-Specific Antibodies in Rheumatoid Arthritis. Int. J. Mol. Sci. 19, (2018).

33. Real-Fernández, F. et al. Surface Plasmon Resonance Method to Evaluate Anticitrullinated Protein/Peptide Antibody Affinity to Citrullinated Peptides. Methods Mol. Biol. 1348, 267-274 (2015).

34. Sakakibara, S. et al. Clonal evolution and antigen recognition of anti-nuclear antibodies in acute systemic lupus erythematosus. Sci. Rep. 7, 16428 (2017).

35. Poulsen, T. R., Jensen, A., Haurum, J. S. \& Andersen, P. S. Limits for antibody affinity maturation and repertoire diversification in hypervaccinated humans. J. Immunol. 187, 4229-4235 (2011).

36. Reddy, S. B. et al. Differences in affinity of monoclonal and naturally acquired polyclonal antibodies against Plasmodium falciparum merozoite antigens. BMC Microbiol. 15, 133 (2015). 
561 37. Pratesi, F. et al. BNT162b2 mRNA SARS-CoV-2 Vaccine Elicits High Avidity and Neutralizing Antibodies in Healthcare Workers. Vaccines (Basel) 9, (2021).

38. Xiong, H., Dolpady, J., Wabl, M., Curotto de Lafaille, M. A. \& Lafaille, J. J. Sequential class switching is required for the generation of high affinity $\lg E$ antibodies. J. Exp. Med. 209, 353-364 (2012).

39. Mita, H., Yasueda, H. \& Akiyama, K. Affinity of IgE antibody to antigen influences allergen-induced histamine release. Clin. Exp. Allergy 30, 1583-1589 (2000).

568

569

570

571

572

573

574

575

576

577

578

579

580

581

582

583

40. Wang, J. et al. Correlation of IgE/lgG4 milk epitopes and affinity of milk-specific IgE antibodies with different phenotypes of clinical milk allergy. J. Allergy Clin. Immunol. 125, 695-702, 702.e1 (2010).

41. Pierson, L., Allauzen, S., Blumenthal, M. \& Rosenberg, A. An automated method for determination of antibody affinity distribution functions with nanogram quantities. $J$. Immunol. Methods 211, 97-109 (1998).

42. Péterfy, F., Kuusela, P. \& Mäkelä, O. Affinity requirements for antibody assays mapped by monoclonal antibodies. J. Immunol. 130, 1809-1813 (1983).

43. Alessi, P., Fermeglia, M. \& Kikic, I. Significance of dilute regions. Fluid Phase Equilib 70, 239-250 (1991).

44. Dimitrov, D. S. Therapeutic antibodies, vaccines and antibodyomes. MAbs 2, 347356 (2010).

45. Prechl, J. Network organization of antibody interactions in sequence and structure space: the RADARS model. Antibodies (Basel) 9, (2020).

46. Prechl, J. Why current quantitative serology is not quantitative and how systems immunology could provide solutions. Biologia Futura (2021) doi:10.1007/s42977-020$00061-1$. 
47. Prechl, J. Quantitative network organization of interactions emerging from the evolution of sequence and structure space of antibodies: the RADARS model. BioRxiv (2018) doi:10.1101/438804.

588

48. De Michele, C., De Los Rios, P., Foffi, G. \& Piazza, F. Simulation and Theory of Antibody Binding to Crowded Antigen-Covered Surfaces. PLoS Comput. Biol. 12, e1004752 (2016).

591

49. Edwards, D. A. Steric hindrance effects on surface reactions: applications to BIAcore. J. Math. Biol. 55, 517-539 (2007). 


\section{Acknowledgments}

598 We thank participants of the GAPAID consortium, especially László Czirják, for providing serum samples for the study and Paolo Rovero for providing VCP2 peptide.

600

601

\section{Author contributions}

602 Á.K. analyzed data, developed model, wrote fitting program

603 J.P. conceptualized of strategy, designed experiments, wrote manuscript, created figures, 604 supervised study

605 J.R. carried out measurements

606 K.L. designed experiments

607 K.P. designed experiments, analyzed data, contributed to figures

608 O.A. carried out measurement, analyzed data, wrote manuscript

609 T.P. conceptualized and developed model

610 Z.H. produced microarrays, carried out measurements

611

612 Conflict of interest

613 K.P., Z.H. and J.P. are employed by Diagnosticum Zrt, a company that provides custom

614 microarray services. A provisional patent application related to this work has been filed by

615 Diagnosticum zrt.

616

617 Funding

618 JP was partially supported by VEKOP-2.1.1-15-2016-00098 grant, part of Széchenyi 2020

619 project, to Diagnosticum. This work of TP was completed in the ELTE Institutional Excellence

620 Program (TKP2020-IKA-05) financed by the Hungarian Ministry of Human Capacities. 


\section{$623 \quad$ Figure legends}

624

625 Figure 1. Simultaneous titration of antigen density and serum antibody

626 Steps of the technology, starting with microarray fabrication and measurement, through image

627 analysis to curve fitting and visualization of results are shown. Serum dilution is indicated by

628 red drops, antigen density differences are represented by shades of blue circles. Fitting data

629 with generalized logistic curves yields two parameters, $x_{i}$ and $d$, which characterize chemical 630 potential distribution of serum antibodies.

631

632 Figure 2. Fitting in two dimensions

633 Examples of parallel measurements of IgG and IgA binding to diagnostic celiac disease peptide

634 epitope are shown, with serum dilutions in three-fold (A) and two-fold (B) steps. Dot symbols

635 stand for measurement data, lines are fitted curves.

636

637 Figure 3. Comparative characterization of serum antibody binding

638 Using the values of the parameters obtained by curve fitting, calculated thermodynamic activity 639 coefficient distributions are comparable not only for serum samples (A) but also for distinct 640 antibody classes (B). Results shown in Figure 1 were used for the generation of normalized

641 comparisons. Bar charts (C) show mean $d$ and $x_{i}$ values, and 95\% confidence intervals as 642 whiskers.

643

644 Figure 4. Conventional titration of serum.

645 Mid-point and end-point titers of $\operatorname{IgA}$ and $\operatorname{IgG}$ are calculated by logistic fitting of binding to 646 distinct antigen densities and are displayed as a function of antigen density.

647

648 Figure 5. Characterization of a monoclonal $\mathrm{Ab}$ for reference.

649 Binding data of monoclonal Ab 14D15 was fitted using the same algorithm as for serum 650 antibodies. Binding data and fitted curves (A), calculated distribution of activity coefficient (B) and estimates of parameters $\mathrm{x}_{\mathrm{i}}$ and $\mathrm{d}(\mathrm{C})$ are shown. 


\section{Symbols and abbreviations}

654

655

a relative thermodynamic activity

A antibody capacity $[\mathrm{FI}]$

$\mathrm{Ab} \quad$ antibody

[Ab] antibody concentration [mol/L]

$\mathrm{AbAg}$ antibody-antigen complex

[AbAg] antibody-antigen complex concentration [mol/L]

Ag antigen

[Ag] antigen concentration $[\mathrm{mol} / \mathrm{L}]$

B auxiliary parameter

BSA Bovine Serum Albumin

c molar concentration $[\mathrm{mol} / \mathrm{L}]$

C auxiliary parameter

d shape parameter that determines asymmetry

D positive constant $[\mathrm{L} / \mathrm{mol}]$

ELISA Enzyme-Linked Immunosorbent Assay

$\gamma \quad$ thermodynamic activity coefficient

IgA immunoglobulin A

IgG immunoglobulin $\mathrm{G}$

$\mathrm{k} \quad$ rate of exponential growth

$\mathrm{K}_{\mathrm{D}} \quad$ equilibrium dissociation constant 
m auxiliary parameter

$\mu \quad$ chemical potential $[\mathrm{J} / \mathrm{mol}]$

$\mu^{0} \quad$ standard chemical potential $[\mathrm{J} / \mathrm{mol}]$

mAb monoclonal antibody

PBS Phosphate-Buffered Saline

$\mathrm{R} \quad$ ideal gas constant $\left[\sim 8.314 \mathrm{~J} / \mathrm{K}^{*} \mathrm{~mol}\right]$

$\mathrm{R}(\mathrm{x}) \quad$ Richards function

RFI Relative Fluorescence Intensity

RIA Radioimmunoassay

s slope of $\ln R(x)$ at minus infinity

$\mathrm{T} \quad$ temperature $[\mathrm{K}]$

$\mathrm{x}_{\mathrm{i}} \quad$ value of $\mathrm{x}$ at inflection point

$\mathrm{y}_{\mathrm{i}} \quad$ value of $\mathrm{y}$ at inflection point

z serum dilution factor [1/dilution] 
658 Figure 1. Simultaneous titration of antigen density and serum antibody

659 Steps of the technology, starting with microarray fabrication and measurement, through image 660 analysis to curve fitting and visualization of results are shown. Serum dilution is indicated by 661 red drops, antigen density differences are represented by shades of blue circles. Fitting data 662 with generalized logistic curves yields two parameters, $x_{i}$ and $d$, which characterize chemical 663 potential distribution of serum antibodies.

664

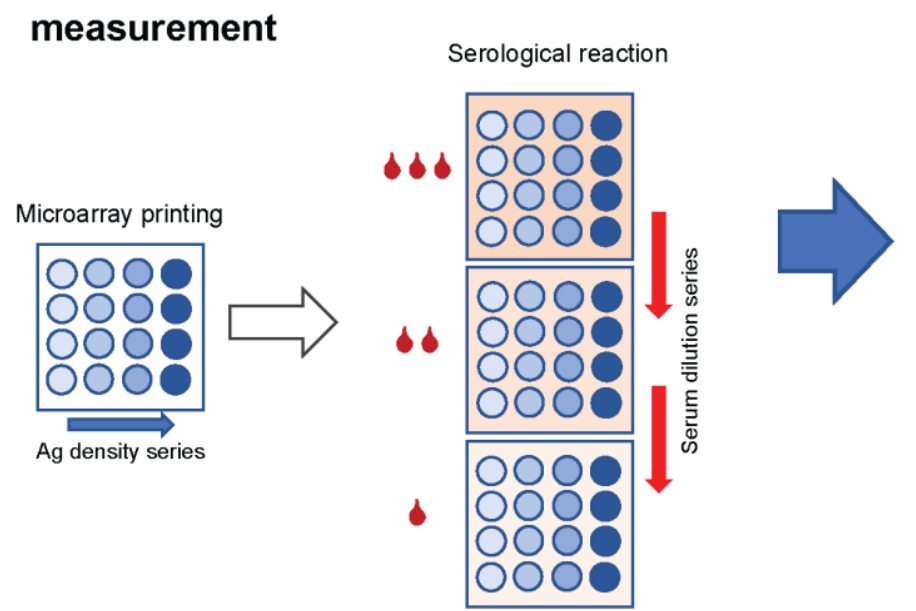

image analysis

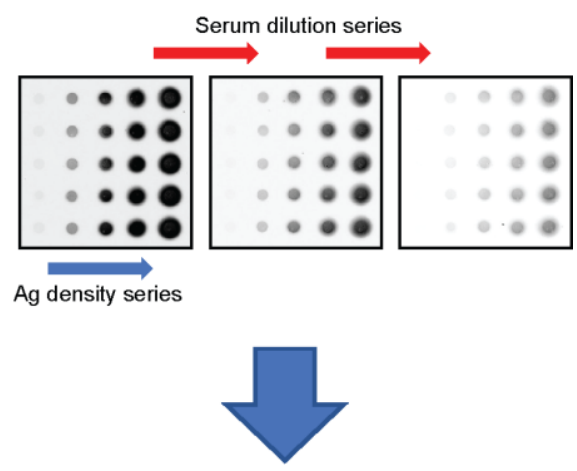

2D fitting
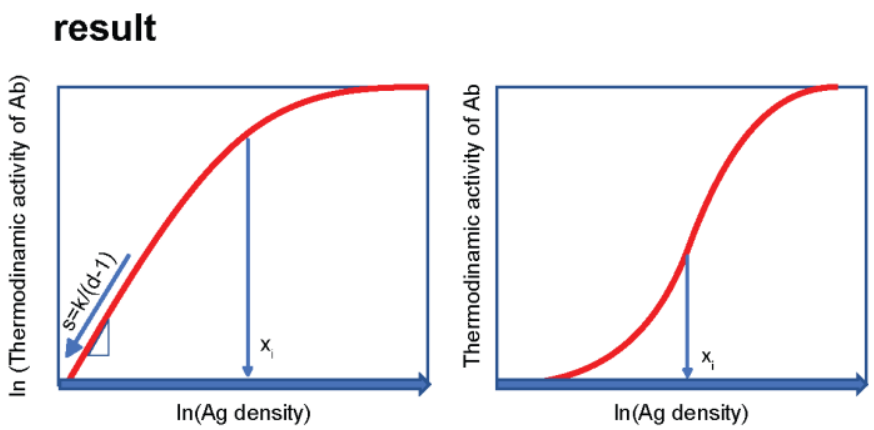

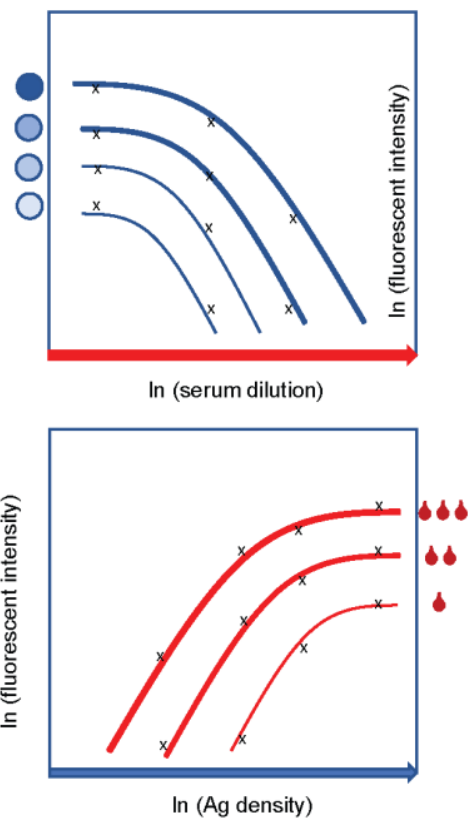


668 Figure 2. Fitting in two dimensions

669 Examples of parallel measurements of IgG and IgA binding to diagnostic celiac disease peptide 670 epitope are shown, with serum dilutions in three-fold (A) and two-fold (B) steps. Dot symbols 671 stand for measurement data, lines are fitted curves, numbers are sample identification numbers.

A
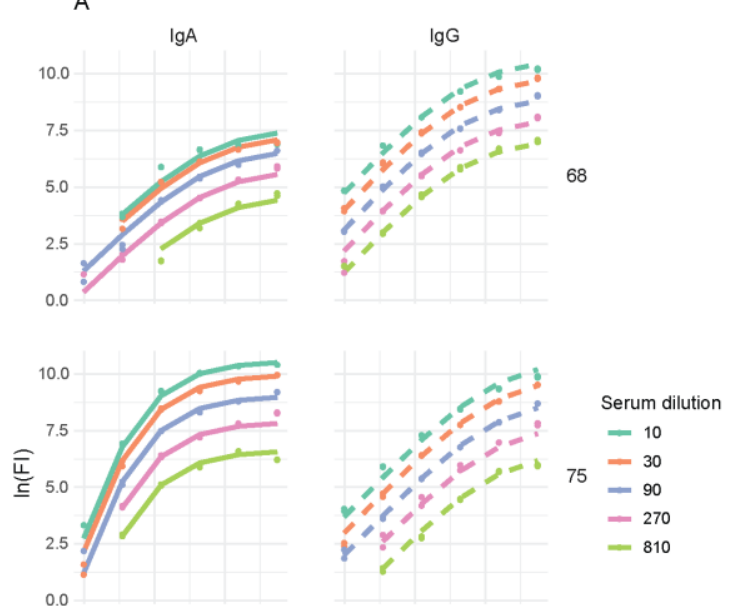

673

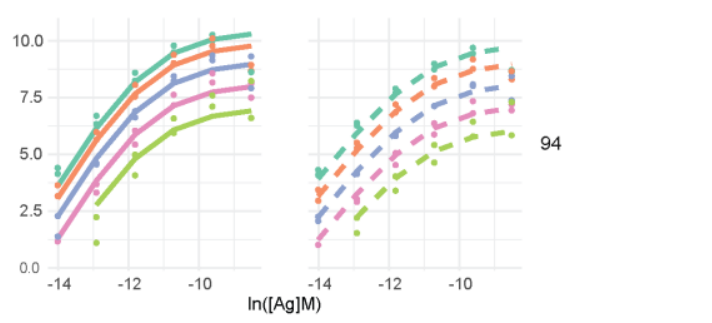

B

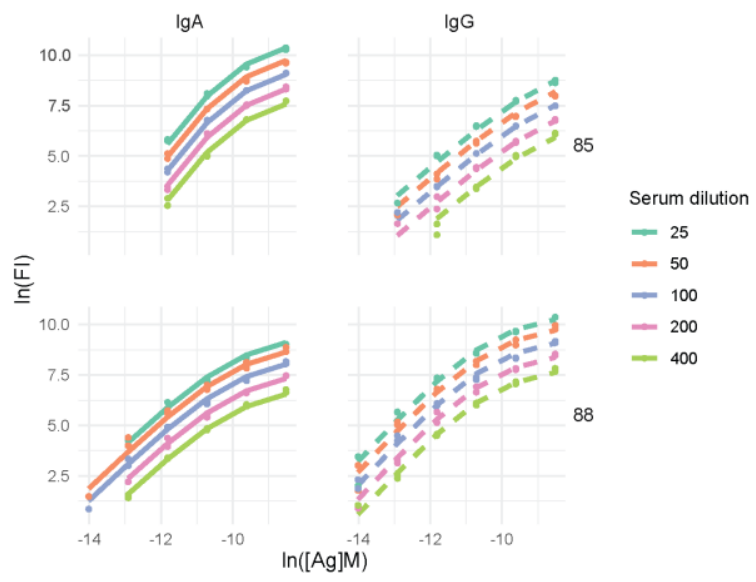


676 Figure 3. Comparative characterization of serum antibody binding

677 Using the values of the parameters obtained by curve fitting, calculated thermodynamic activity 678 coefficient distributions are comparable not only for serum samples (A) but also for distinct 679 antibody classes (B). Results from data shown in Figure 2 were used for the generation of 680 normalized comparisons. Bar charts (C) show mean $d$ and $x_{i}$ values, and $95 \%$ confidence 681 intervals as whiskers.

682

A

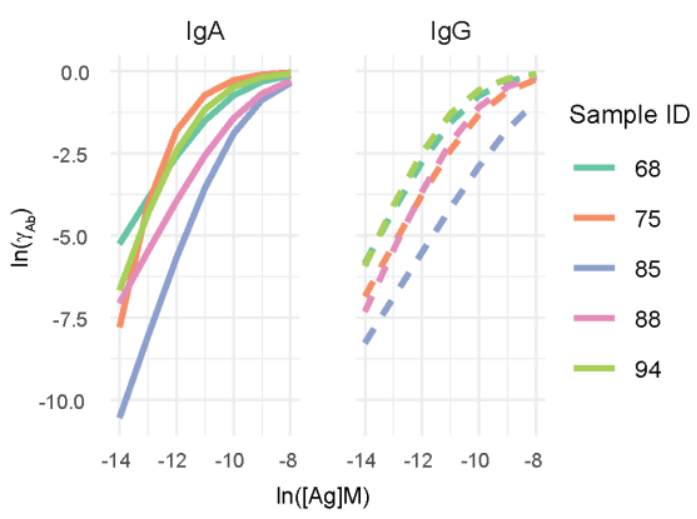

C

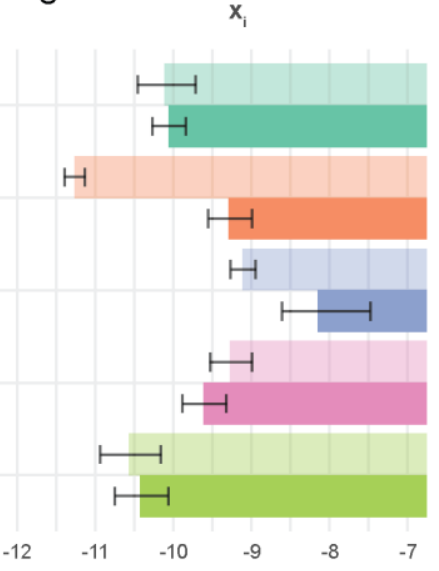

B

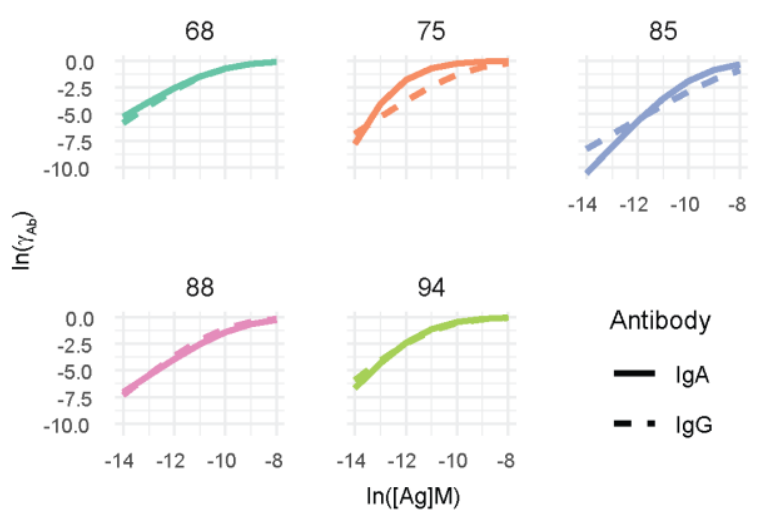

d

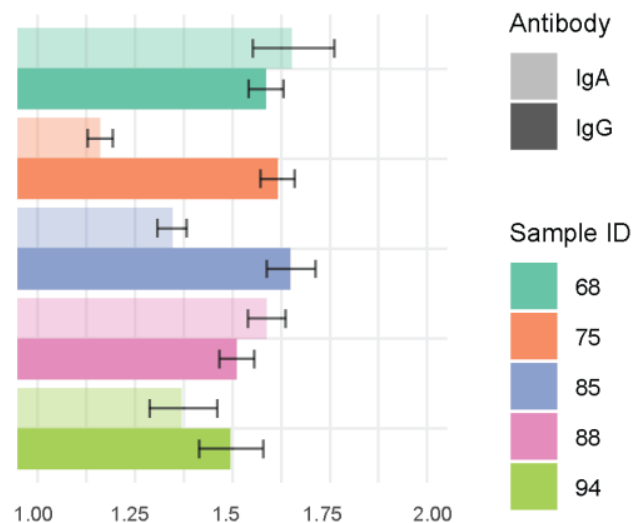


686 Figure 4. Conventional titration of serum.

687 Mid-point and end-point titers of $\operatorname{IgA}$ and $\operatorname{IgG}$ are calculated by logistic fitting of binding to 688 distinct antigen densities and are displayed as a function of antigen density.

689
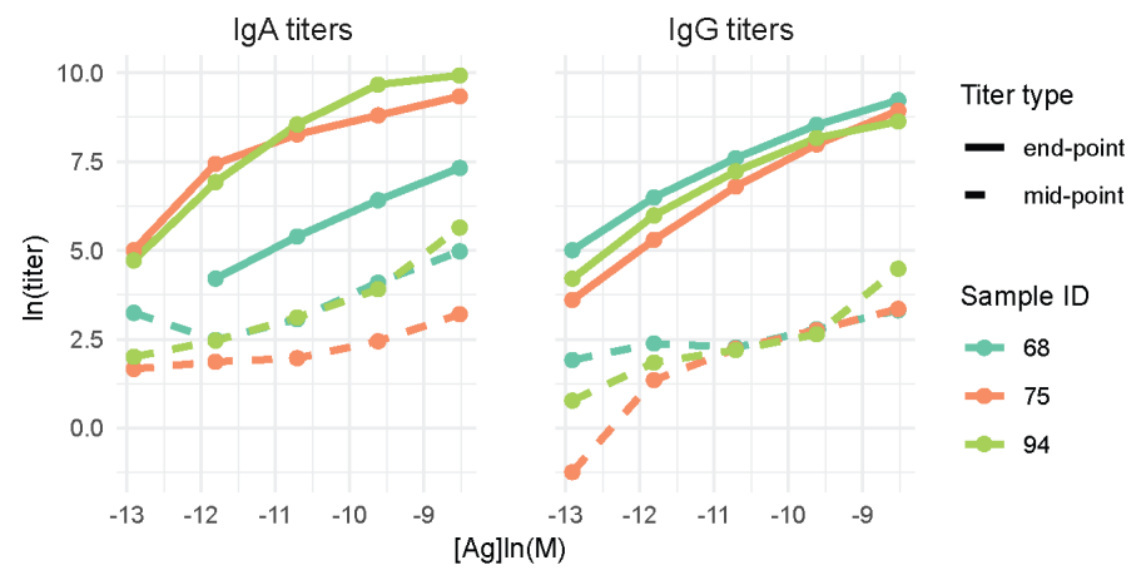
693 Figure 5. Characterization of a monoclonal $\mathrm{Ab}$ for reference.

694 Binding data of monoclonal Ab 14D15 was fitted using the same algorithm as for serum 695 antibodies. Binding data and fitted curves (A), calculated distribution of activity coefficient (B) 696 and estimates of parameters $\mathrm{x}_{\mathrm{i}}$ and $\mathrm{d}(\mathrm{C})$ are shown.

697

A

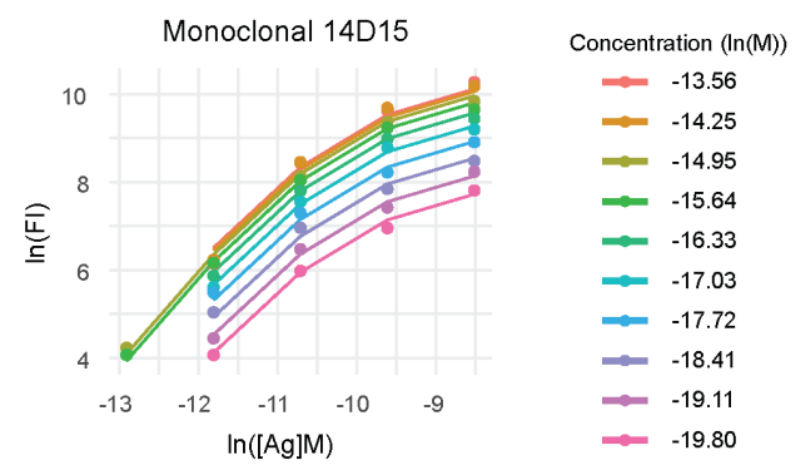

B

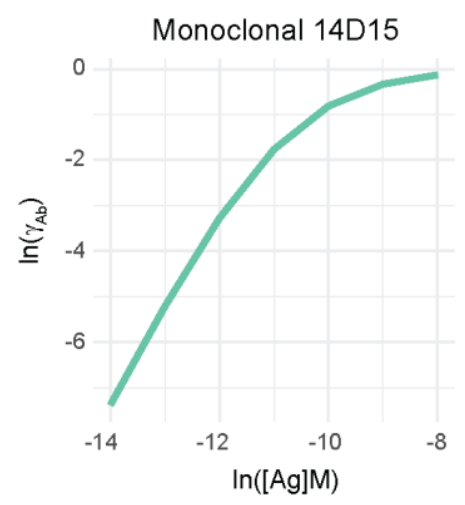

C

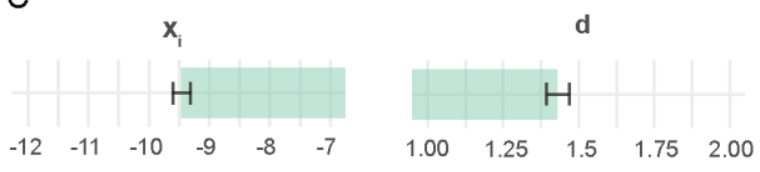

